\title{
Myofascial Trigger Points and the Body Posture - Deactivating them Applying Magnetic Fields (preliminary reports)
}

\author{
J. JutrzenkA-Jesion ${ }^{a, *}$, M. Chochowska ${ }^{b}$ And D. HojAn-Jezierska ${ }^{a, c}$ \\ ${ }^{a}$ Stanisław Staszic State School of Higher Vocational Education in Piła \\ ${ }^{b}$ College of Education and Therapy in Poznań \\ ${ }^{c}$ Poznań University of Medical Sciences
}

\begin{abstract}
Myofascial pain syndrome is sensory, motor, autonomic disorder caused by the occurrence of trigger points $(\operatorname{TrP})$. In literature the possibilities of deactivating TrPs through exposure to magnetic field (MF) can be noticed. The aim of current research is an attempt at answering the question whether MF changes TrP activity. Tests were carried out on 22 volunteers, who were divided into three groups. First was exposed to static MF using MagneticUnit discs, second was exposed to slow changing MF using Viofor-JPS. The third was exposed to magnetoledotherapy, also using Viofor-JPS.
\end{abstract}

DOI: 10.12693/APhysPolA.125.A-16

PACS: $87.50 . \mathrm{C}-$

\section{Introduction}

Myofascial pain syndrome (MPS) is defined as sensory, motor and autonomic disorder caused by the occurrence of trigger points (TrP) [1]. Travell and Simons define $\mathrm{TrP}$ as hyperirritable spot within skeletal muscle, linked to a nodule that was hyper irritable during palpation and which can be found in a too taut muscle band. The spot is painful when compressed and may cause symptoms characteristic for referred pain, hyperirritability, motor dysfunctions and autonomic symptoms [2]. According to other authors $\operatorname{TrP}$ are very sensitive areas within hypertonic tissues, which may occur not only in myofascia but also periosteum and other connective tissue structures [3].

Compression or other form of irritation applied to $\operatorname{Tr} \mathrm{P}$ (e.g. needling) causes local, radiating or referred pain as well as other symptoms (pins and needles, numbness, burning and itching) in areas often very distal from the place of application $[1,2,4,5]$.

Poor and asymmetric body posture leads to increasing tension in individual groups of tissues. When the situation is prevailing for long then oxygen level in tissues decreases. In case of oxygen shortage production of energy requires the process of anaerobic glycolysis. It results in increasing the concentration of the products of anaerobic glycolysis: lactic and pyruvic acid [6]. At compromised, due to increased muscle tension, blood circulation, growing concentration of those substances is manifested through pain and discomfort. Pain and discomfort increase muscle tension even further, irritating local nocyceptors, resulting in

*corresponding author; e-mail: joannajutrzenka@wp.pl progressing tissue irritability [7]. It triggers the process of tissue degeneration during which transverse bridges are built lowering elasticity; in the long run those tissues become fibrotic and sclerotic. Modifications in the process of protein synthesis occur which slows down healing processes. The process of TrP building begins, causing further disorders in body asymmetry [8]. A mechanism of vicious circle, described by the sequence: posture asymmetry $\Rightarrow$ hypertonic tissues $\Rightarrow$ decrease of oxygen level in tissues $\Rightarrow$ anaerobic glycolisis $\Rightarrow$ concentration of lactic and pyruvic acid $\Rightarrow$ pain $\Rightarrow$ increase in tension $\Rightarrow$ irritation of nerve endings $\Rightarrow$ sensitizing tissues $\Rightarrow$ tissues degeneration $\Rightarrow$ modification of the process of protein synthesis $\Rightarrow$ development of $\operatorname{TrPs} \Rightarrow$ further intensification of posture asymmetry is created.

There are numerous methods of deactivating $\operatorname{TrP}$. Among popular ones there are: manual therapy, dry needling, physical therapy (e.g. ultrasounds, laser therapy, electrotherapy, magnetotherapy) kinesiology taping and massage [8, 9].

The study presents an attempt at deactivating $\operatorname{Tr} P$ using low-frequency and static magnetic field (MF) also combined with infrared light.

In magnetostimulation is used magnetic fields with frequency $2000-3000 \mathrm{~Hz}$ and induction 1-100 $\mu \mathrm{T}$ (induction closely proportional to that in earth's magnetic field) [12].

The impact of low-frequency MF on living organisms it's possible to explain through resonance mechanisms and embraces the following effects: anti-inflammatory, anti-oedematous and analgesic [10-13]. They also intensify the process of cellular respiration, as well as the processes of trans-membrane transport and soft tissues regeneration. It's vasodilating and angiogenic effect. What also seems significant is the fact that its analgesic effect 
occurs not only during the exposure to MF but also following the completion of magneto-stimulation. It proves biological hysteresis of MF effect.

While magnetoledotherapy is a form of therapy that combines simultaneous effect of two physical factors that is MF and infrared light. Using magnetostimulation combined with light one may expect synergy of both types of electromagnetic radiation in a form of changes in activity of serotonin synapses and the intensification of $\beta$-endorphin secretion. Also the stimulation of pituitaryadrenal axis can be expected along with the increase in glucocorticoids secretion resulting in enhancing analgesic effect. [8, 10-12, 14].

Application of static MF in the treatment of different disorders has been proved by the results of clinical research [8, 15-18, 21]. Of special significance is its analgesic effect combating pain of various etiology $[8,15,18-22]$. The effect that static MF has on living organisms is the result of that field on uncompressed electron spins, diamagnetic molecules and dynamic electric charges $[11,14,20]$.

The aim of the study presented in the article was an attempt at answering the question if and how the presence of $\operatorname{TrP}$ influences body arrangement in space - that is body posture as well as an attempt at deactivating TrPs using static and low-frequency MF.

\section{Experimental}

The patients were examined five times: prior to the beginning of therapy, two and four weeks after the therapy as well as two and four weeks following the completion of treatment. During each examination palpation tenderness of $\mathrm{TrP}$ was measured- using VAS scale. All patients prior to the therapy were examined using Zebris PDM platform.

Initial analysis of study results shows the efficacy of all three forms of application. The best result has been achieved using Viofor (avg.: 6.67 at the beginning of therapy vs. 1.5 at the end using VAS scale) and MagneticUnit (avg.: 6.5 at the beginning of therapy vs. 2 at its end using VAS scale).

To evaluate body posture and the presence of possible asymmetric loads DM-S Zebris platform was used. It facilitated static and dynamic measure of weight loading during walking and in standing. It consists of active measuring space made of 2650 receptors recording weight loading on the base measuring $34 \times 54 \mathrm{~cm}^{2}$ [19]. Selected for the study static module of the available software allows for the analysis of force distribution through feet on the ground in standing (test period: 30 seconds) as well as balance evaluation, through tracking the location of the centre of feet pressure on the platform and the asymmetry of placing feet and loading them (right foot vs. left foot; forefoot vs. Heel). Exemplary report was presented in Fig. 1.

Group participating in the study consisted of 22 volunteers aged 20-30. Among them there were women and

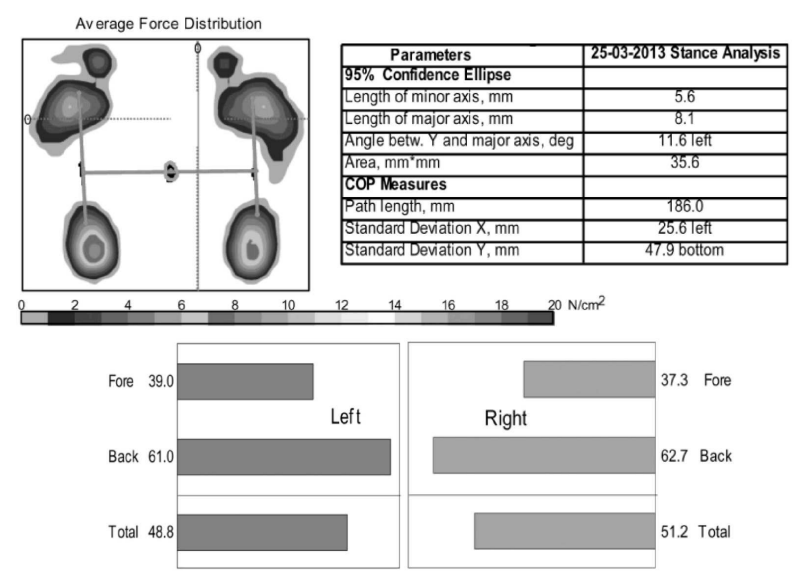

Fig. 1. Exemplary report - average force distribution through feet.

men unaffected by other disorders (active cancer, tuberculosis, bleeding in the digestive track, acute virus, bacterial or mycotic infections, presence of electronic implants, conditions following organ transplants) not pregnant and declaring good general health condition. None of the participants had any contraindications for either magnetostimulation, magnetoledotherapy or magnetotherapy.

The participants were divided into three groups:

Group I: was exposed to magnetoledostimulation with the following parameters: low-frequency MF, VIOFORJPS clinic apparatus, program-P3, application methodM2, intensity-6, infrared applicator (IR), stimulation period $36 \mathrm{~min}$, every day for 2 weeks, followed by one week break and two more weeks of stimulation.

Group II: was exposed to magnetic stimulation with the following parameters: low-frequency MF, VIOFOR-JPS clinic apparatus, medium applicator ("pillow") programP3, application method-M2, intensity-6, small applicator, stimulation period $36 \mathrm{~min}$, every day for 2 weeks, followed by one week break and two more weeks of stimulation.

Group III: was exposed to static gradient field using MagneticUnit discs (2 elements), stimulation period $30 \mathrm{~min}$, every day for 4 weeks.

Prior to the beginning of stimulation using MF each participant underwent palpation test of the following muscles: trapezius, latissimusss dorsi, erector spinae, quadriceps femoris, tensor facia lata, supraspinatus and sartorius - to verify the presence of TrPs.

Tests were bilateral. Localized $\operatorname{TrP}$ was clearly marked to make sure that in the following tests the same spots were checked. All TrPs exposed to therapy were latent.

The next step was the examination of tenderness of the TrPs found under pressure and patient's subjective evaluation (according to patient's feeling) of the level of tenderness to pressure using 10 point-Visual Analogue Scale (VAS), where "0" indicated lack of pain and "10" unbearable pain. TrP tenderness tests applying pressure were used five times: 1 ) prior to the beginning of therapy, 
2) after two weeks of therapy; 3) directly following the completion of therapy (after 4 weeks), and then two and four weeks following the end of therapy (tests 4 and 5).

Moreover, each participant filled in author's questionnaire and underwent force distribution test through feet in standing (barefooted on FDM-S Zebris platform). That test on Zebris FDM platform was carried out once prior to the commencement of the study.

\section{Results and Discussion}

Results obtained on FDM-S Zebris platform showed that loading through feet in individual patients ranged from 6 to $20 \mathrm{~N} / \mathrm{cm}^{2}$. In case of 17 participants the results fell within the accepted norms [19], that is they did not exceed $15 \mathrm{~N} / \mathrm{cm}^{2}$. While 5 patients presented excessive ground loading.

Only 2 patients showed balanced force distribution through both sides of their body. 14 patients showed excessive loading through right side of their body and 6 left side.

In case of excessive loading through forefoot only two patients obtained results that fell within the accepted range which is $33 \%$ (forefoot vs. heel). The next 12 participants excessively loaded forefoot: seven of them obtained results exceeding $43 \%$ of their body weight. 8 of the participants insufficiently loaded through forefoot - carrying on that part of their feet the weight lower than the one indicated in norms (below $33 \%$ of body weight). Correct loading through heel was recorded in case of only one patient while the other 12 participants obtained results below the norm and 9 excessively loaded their heel (the results refer to left foot).

TABLE I

Trigger points tenderness test in three groups embraced by the study.

\begin{tabular}{c|c|c|c|c}
\hline \hline \multirow{2}{*}{ Test $^{*}$} & \multicolumn{4}{|c}{ VAS scale (average) } \\
\cline { 2 - 5 } & Group I & Group II & Group III & Group I-III \\
\hline 1 & 7.1 & 6.7 & 6.5 & 6.8 \\
\hline 2 & 3.6 & 3.7 & 3.6 & 3.6 \\
\hline 3 & 2.5 & 1.5 & 2 & 2 \\
\hline 4 & 2.8 & 2 & 1.4 & 2 \\
\hline 5 & 2.8 & 2 & 2 & 2.3 \\
\hline
\end{tabular}

*1 - before therapy, 2 - after two weeks of therapy, 3 - after four weeks of therapy, 4 - after two weeks from the end of therapy, 5 - after four weeks from the end of therapy

Average tenderness of tested TrP (VAS scale) resulted in all three groups as follows: I -7.1 ; II -6.8 ; III -6.5 (av. tenderness in all groups - 6.8). After two weeks of using magnetosymulation the level of tenderness fell to: I -3.6 ; II -3.7 ; III -3.6 (av. tenderness in all groups - 3.6). After four weeks of therapy: I - 2.5; II 1.5; III - 2 (av. tenderness in all groups - 2). The analgesic effect remained after completion of tests. After two weeks from the of therapy, tenderness amounted to
TABLE II

Statistical differences among following researches in each tested group.

\begin{tabular}{l|c|c|c}
\hline \hline \multirow{2}{*}{ Test* } & \multicolumn{3}{|c}{ Wilcoxon Test $\left(\mathrm{p}^{* *}\right)$} \\
\cline { 2 - 4 } & Group I & Group II & Group III \\
\hline 1 vs. 2 & $\underline{0.012}$ & 0.12 & $\underline{0.012}$ \\
\hline 1 vs. 3 & $\underline{0.017}$ & $\underline{0.028}$ & $\underline{0.012}$ \\
\hline 1 vs. 4 & $\underline{0.012}$ & $\underline{0.028}$ & $\underline{0.012}$ \\
\hline 1 vs. 5 & $\underline{0.012}$ & $\underline{0.046}$ & $\underline{0.012}$ \\
\hline *1 - before therapy, $2-$ after two weeks of therapy, \\
3 - after four weeks of therapy, 4 -after two weeks \\
from the end of therapy, $5-$ after four weeks from \\
the end of therapy; **the underlined results differ sta- \\
tistically significant at the $p<0.05$
\end{tabular}

TABLE III

Statistical differences among tested groups.

\begin{tabular}{c|c|c|c}
\hline \hline \multirow{2}{*}{ Test* $^{*}$} & \multicolumn{3}{|c}{ Mann-Whitney Test (p) } \\
\cline { 2 - 4 } & Grp. I vs. II & Grp. I vs. III & Grp. II vs. III \\
\hline 1 & 0.544 & 0.332 & 0.791 \\
\hline 2 & 0.691 & 0.629 & 0.600 \\
\hline 3 & 0.367 & 0.957 & 0.552 \\
\hline 4 & 0.294 & 0.102 & 0.420 \\
\hline 5 & 0.511 & 0.593 & 0.947 \\
\hline
\end{tabular}

*1 - before therapy, 2 - after two weeks of therapy, 3 - after four weeks of therapy, 4 - after two weeks from the end of therapy, 5 - after four weeks from the end of therapy

: I - 2.8; II -2 ; III -1.4 (av. tenderness in all groups - 2). After four weeks from the end of therapy, tenderness amounted to : I -2.8 ; II -2 ; III -2 (av. tenderness in all groups - 2.3) - Table II. The described results were statistically significant (Wilcoxon Test, $p<$ $0.05)$ - Table III. There were no statistically significant differences in the results achieved in each tested group (Mann-Whitney Test, $p<0.05$ ) — Table IV.

\section{Conclusions}

1. The above results show that the majority of the patients who underwent tests were characterized by imbalanced body posture manifested by inadequate feet loading outside the accepted norms of static force distribution through feet (right vs. left foot; forefoot vs. heel), what might be caused, according to the available literature, by the presence of latent TRPs.

2. Simultaneously the other half of those participating in the study pointed at a significant efficacy of magnetostimulation (both using static and lowfrequency MF) when deactivating TrPs.

3. The best effect was obtained in groups II and III - where Viofor apparatus was used (avg.: at the 
beginning of therapy 6.7 in VAS vs.1.5 following the completion of therapy) and MagneticUnit (avg.: at the beginning of therapy 6.5 in VAS vs. 2 following its completion), the least significant improvement was recorder in group I, where magnetoledotherapy was used (avg.: at the beginning of therapy 7.1 in VAS vs. 2.5 following its completion).

4. It should be emphasized that analgesic effect of the MF used remained also following the completion of therapy and decreased TrPs tenderness was kept on low level even four weeks from the completion of stimulation using MF.

\section{References}

[1] M. Chochowska, M. Wytrążek, J.T. Marcinkowski, Physiotherapy 20, 89 (2012).

[2] J. Travell, D.G. Simons, Myofascial pain and dysfunction. The trigger point manual, Vol. I, Upper half body, Wiliams\&Wilkins, Baltimore 1999.

[3] J. Stodolny, Lumbar Spine Strain - Epidemic of Our Times, ZL Natura, Kielce 1999, (in Polish).

[4] P. Richter, E. Hebgen, Trigger Points and Muscle Chains In Osteopathy and Manual Therapy, Galaktyka, Łódź 2010, (in Polish), p. 5.

[5] L. Chaitow, S. Fritz, A Massage Therarapist's guide to understanding, locating and treating myofascial trigger points, Elsevier Urban\&Partner, Wrocław 2010, (in Polish).

[6] J. Pryor, S. Prassad, Physiotherapy for respiratory and cardiac problems, Churchill Livingstone, Edinburgh 2002.

[7] I.M. Korr, The Neurobiologic Mechanisms in Manipulative Therapy, Springer 1978.
[8] J.S. Janicki, Pract. Rehabil. 2, 15 (2009).

[9] A. Paczesniak-Jost, A. Samojedna-Kobosz, M. Skrzypiec, K. Walicka-Cupruś, I. Opalińska, Young Sport Sci. Ukr. 3, 184 (2011).

[10] M. Woldańska, J. Czernicki, Med. News 58, 44 (2004).

[11] J. Taradaj, A. Sieron, M. Jarzebski, Physical therapy in Practice, Elamed, Katowice 2010, (in Polish).

[12] M. Woldańska-Okońska, Pract. Rehabil. 2, 29 (2009).

[13] D. Hojan-Jezierska, M. Pankowska, A. Wicher, E. Szymiec, M. Kraśnik, M. Knapkiewicz, Acta Phys Pol A 12, A96 (2012).

[14] A. Sieroń, Magnetic Field Therapy in Medicine, Alfamedica, Bielsko-Biała 2002, (in Polish).

[15] W. Samborski, A. Kołaczewska, Medical News 64, 45 (1993).

[16] F. Jaroszyk, Biological Impact of Static Magnetic Fields, Wydawnictwo Akademii Medycznej w Poznaniu, Poznań 1992, (in Polish).

[17] J.S. Janicki, Pract. Rehabil. 4, 15 (2009).

[18] J.S. Janicki, Patient's News 40, (11)40 (2008).

[19] WinFDMS 0.1.x for Windows, rev. 02, 2007.

[20] J.S. Janicki, Ł.J. Janicki, Acta Bio-Opt. Inf. Med. 4, 127 (2008)

[21] A. Sieroń, T. Biniszkiewicz, K. Sieroń, M. Głowacka, K. Biniszkiewicz, Acta Bio-Opt. Inf. Med. 4, 133 (1998).

[22] M. Woldańska-Okońska, J. Czernicki, Acta Bio-Opt. Inf. Med. 8, 5 (2002). 